\title{
Electro-optical modulation with silicon microspheres in liquid crystal
}

\author{
Huzeyfe Y1lmaz and Ali Serpengüzel \\ Koç University, Microphotonics Research Laboratory, Department of Physics, \\ Rumelifeneri Yolu, Sarıyer 34450 Istanbul, Turkey
}

\begin{abstract}
We demonstrate electro-optical tuning and modulation of the optical resonances of a silicon microsphere placed on an optical fiber half coupler and immersed in a nematic liquid crystal. The relative refractive index between the microsphere and the liquid crystal is controlled by an applied external AC electric field. The tuning and modulation of the microsphere optical resonances is monitored with the transmission and elastic scattering signals.
\end{abstract}

Keywords: morphology dependent resonance, whispering gallery mode, coupler, tuning, modulation, optical fiber, silicon, microsphere, liquid crystal.

\section{INTRODUCTION}

Optical microcavities such as microspheres, microtoroids, microdisks and microrings are widely used for emission, modulation, switching, detection and sensing of light because they can confine light for a certain amount of time by total internal reflection, and provide very high quality factor (Q-factor) morphology dependent resonances (MDR's) in very small volumes [1-5]. These high Q-factor MDR's are explained well by the Lorenz Mie theory, and are also called whispering gallery modes (WGM's), in analogy with the whispering acoustic modes of a dome [6]. The MDR's of a microsphere with a radius $a$ and a refractive index $n$, inside a medium with refractive index $n_{l}$ are excited at specific values of the size parameter $x$

$$
x=2 \pi a n_{1} / \lambda
$$

MDR's can be identified with three (polar, radial and azimuthal) mode numbers. In the case of a microsphere, the azimuthal modes are degenerate. The spectral distance between two consecutive polar angular modes, having the same radial mode order, is the mode spacing and can be calculated as follows:

$$
\Delta \lambda=\frac{\lambda^{2} \arctan \sqrt{m^{2}-1}}{2 \pi a n_{1} \sqrt{m^{2}-1}},
$$

where $m$ is the relative refractive index $\left(m=n / n_{l}\right)$ of the microsphere. MDR's of microspheres have been extensively studied in air, water and buffer solutions for sensing applications [7-9], and the shift the MDR's with molecular particle adsorption, mechanical stress, chemical etching and electro-optical effects have been demonstrated. The microsphere MDR's can be tuned by changing the refractive index of the surrounding medium, since this effectively changes the refractive index of the microsphere [10-13].

In addition, nematic liquid crystals (NLC's) are excessively used in display technologies, because of their high birefringence and the ability to reorient under an applied electric field, which makes them perfect candidates as a surrounding medium to tune the MDR's of a microsphere [14].

Here, we demonstrate the tuning and modulation of a silicon microsphere MDR's, observed in the transmission and the $90^{\circ}$ elastic light scattering signals, by applying an external AC electric field to the NLC.

Integrated Photonics: Materials, Devices, and Applications, edited by Ali Serpengüzel, Giancarlo C. Righini, Alfred Leipertz, Proc. of SPIE Vol. 8069, 80690L · @ 2011 SPIE · CCC code: 0277-786X/11/\$18 · doi: 10.1117/12.887572 


\section{EXPERIMENTAL SETUP}

Figure 1 shows the experimental setup. The light at $1.3 \mu \mathrm{m}$, i.e., in the original band (o-band) of optical fiber communications, from a distributed feedback (DFB) semiconductor diode laser is first coupled to the single mode fiber (SMOF). The wavelength and the power of the diode laser are controlled by the laser diode controller. This light is evanescently coupled from the $\mathrm{OFHC}$ to the silicon microsphere with a radius of $500 \mu \mathrm{m}$, which is placed in a nematic liquid crystal (NLC). A signal generator is used to apply an external AC electric potential (hence an external AC electric field) to the NLC. The $90^{\circ}$ elastic scattering from the silicon microsphere is then collected with a standard optical microscope, and detected with an InGaAs photodiode (PD), whose output is fed to a lock-in amplifier. The transmitted light is measured with an InGaAs power wave head (PWH) and read from the optical multimeter (OMM).

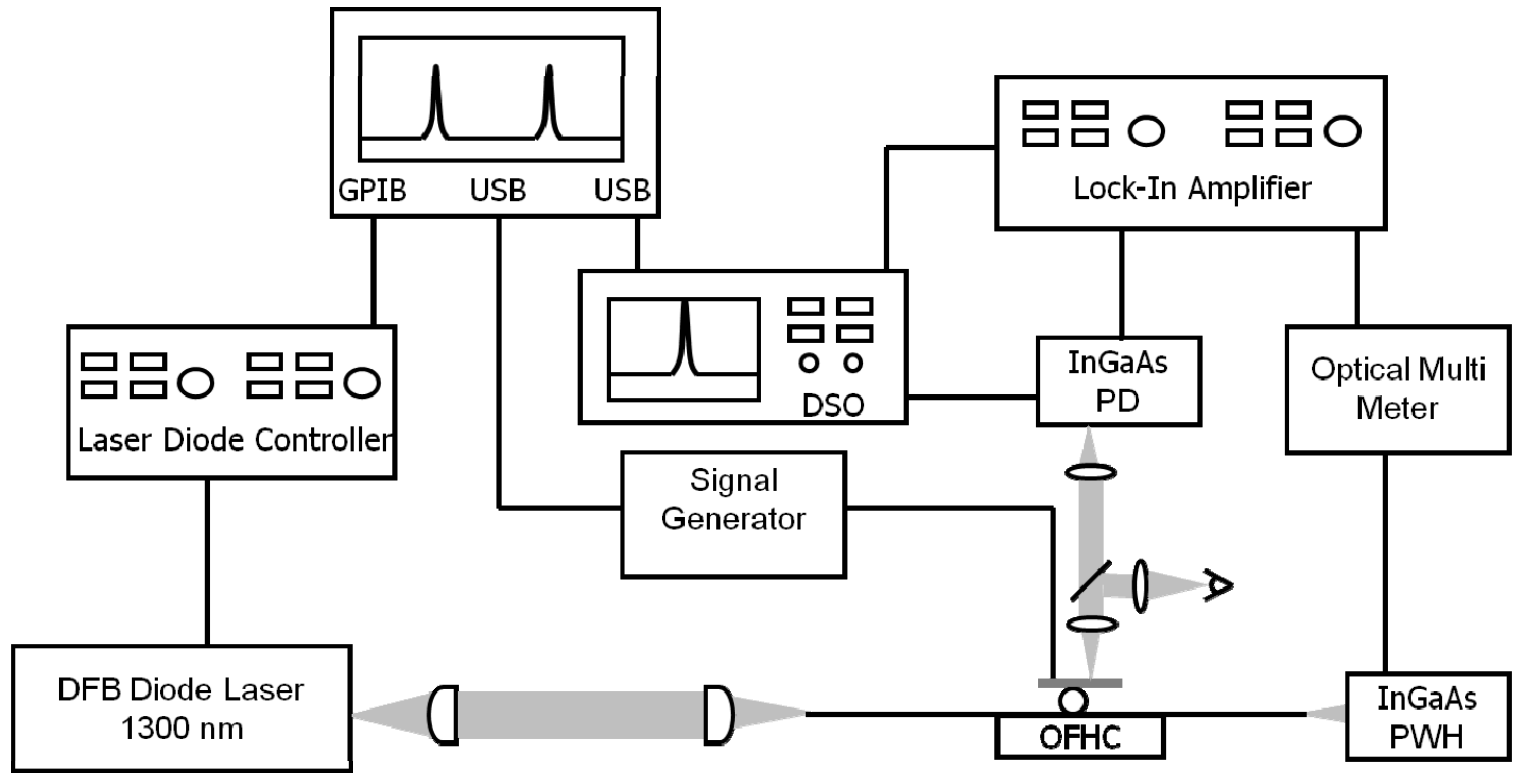

Figure 1. The schematic of the experimental setup.

The NLC used in this experiment is the positively birefringent 4-Cyano-4'-pentylbiphenyl (5CB), with a birefringence of $\Delta \mathrm{n}=0.16$. Both the extraordinary and ordinary refractive indices of the $5 \mathrm{CB}$ are comparable to the refractive indices of the fused silica single mode optical fiber (SMOF). Therefore in the experiment, the NLC is allowed to only cover the top portion of the silicon microsphere.

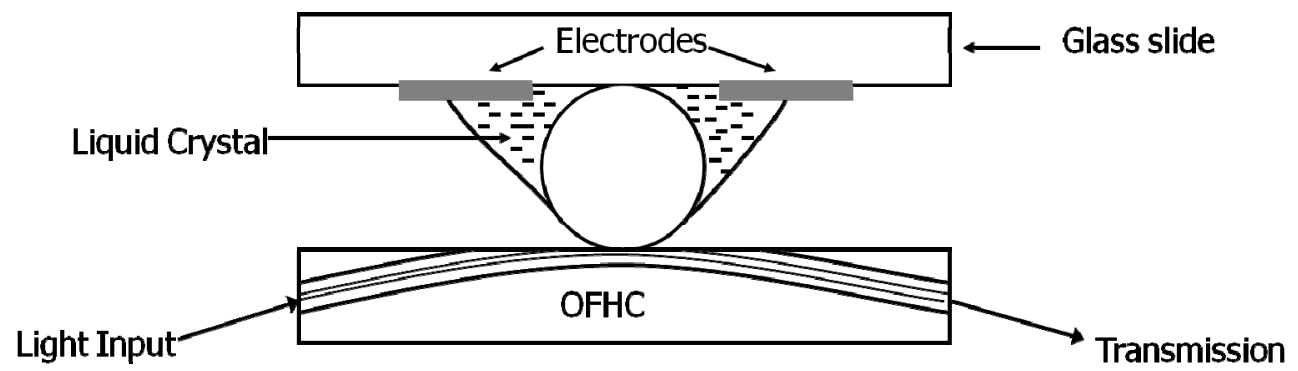

Figure 2. Side view of the silicon microsphere in $5 \mathrm{CB}$ on the OFHC.

Figure 2 shows the schematic of the side view of the silicon microsphere on the OFHC and in the NLC. An external AC electric field is applied to the electrodes to change the relative refractive index of the silicon microsphere and tune its MDR's. 


\section{EXPERIMENTAL RESULTS}

Figure 3 shows the transmission spectra of the silicon microsphere. The dips in the transmission spectrum correspond to the MDR's of the silicon microsphere. In the upper curve, a continuous waveform (CW) alternating current (AC) electric field (E) with a root-mean-square (RMS) value of $0.4 \mathrm{~V} / \mu \mathrm{m}$ is applied to NLC. In the lower curve, no external electric field is applied to the NLC. When the CW external AC electric field is applied to the microsphere, the transmission spectrum shows a red shift of $0.014 \mathrm{~nm}$, corresponding to a change of $10^{-3} \%$.

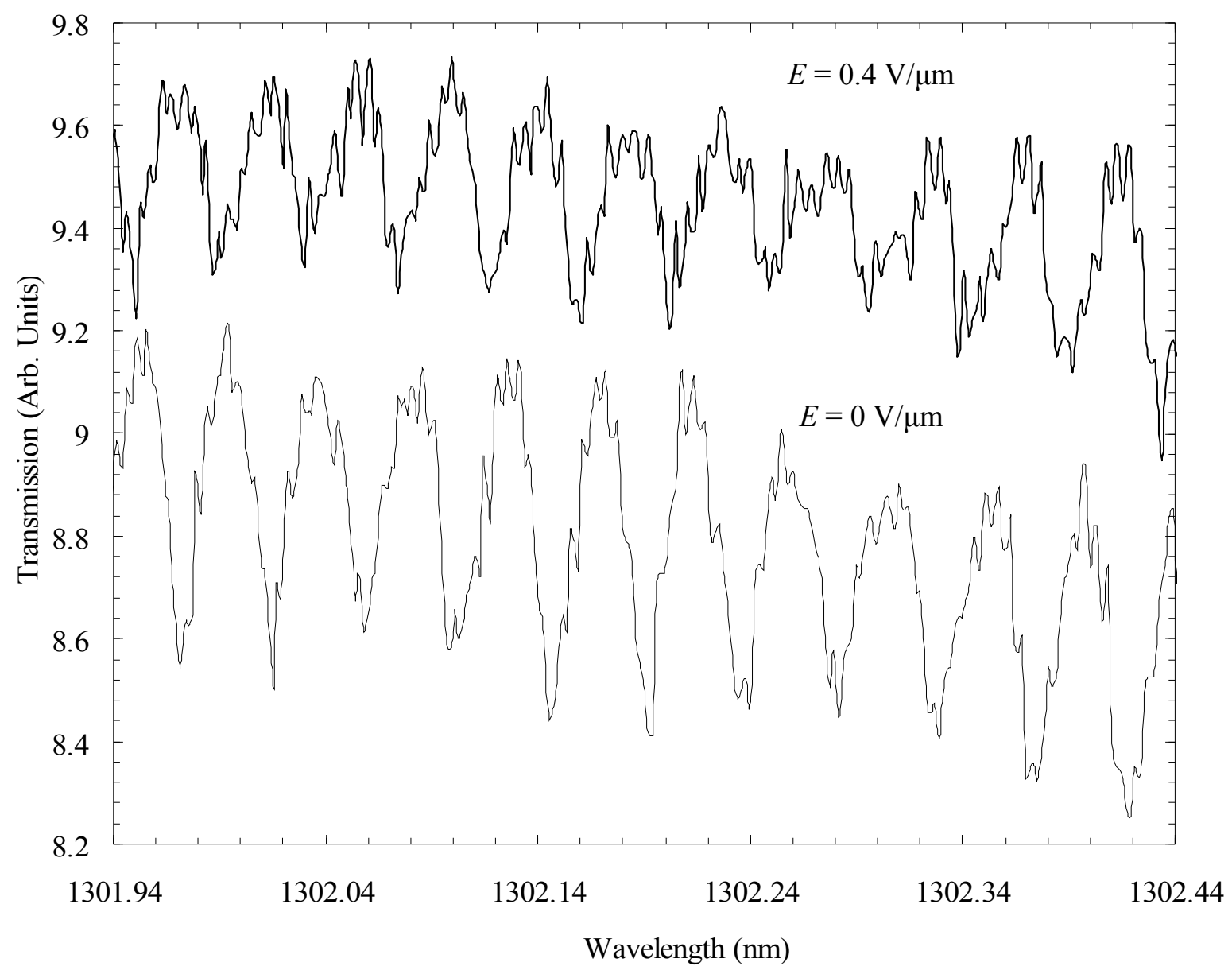

Figure 3. Transmission spectra of a silicon microsphere on the OFHC and in the NLC with (upper curve) and without (lower curve) an applied AC electric field.

When there is no applied external AC electric field, the NLC molecules are in their rest state. As the external AC electric field is applied, the NLC molecules are expected to orient along the direction of the external AC electric field. The silicon microsphere might also heat up due to an induced $\mathrm{AC}$ current. These effects result in the alteration of the relative refractive index of the silicon microsphere and the shift of the elastic scattering and the transmission spectra. This spectral shift gives the opportunity to modulate the amplitude of the elastically scattered and the transmitted light signals from the silicon microsphere, when operating at a constant laser wavelength.

Applying a modulated external $\mathrm{AC}$ electric field is expected to result in the modulation of the relative refractive index and a modulation of the $90^{\circ}$ elastic scattering and the transmitted light intensities from the silicon microsphere. To test this hypothesis, a modulated AC electrical signal is applied to the NLC, as shown in the lower curve of Figure 4. The modulation signal of the AC electric field is at $7 \mathrm{~Hz}$, whereas the carrier signal is at $234 \mathrm{~Hz}$. The upper curve of Figure 4 
shows the measured $90^{\circ}$ elastic scattering intensity, exhibiting a modulation, which is in phase with the modulating external AC electric field.

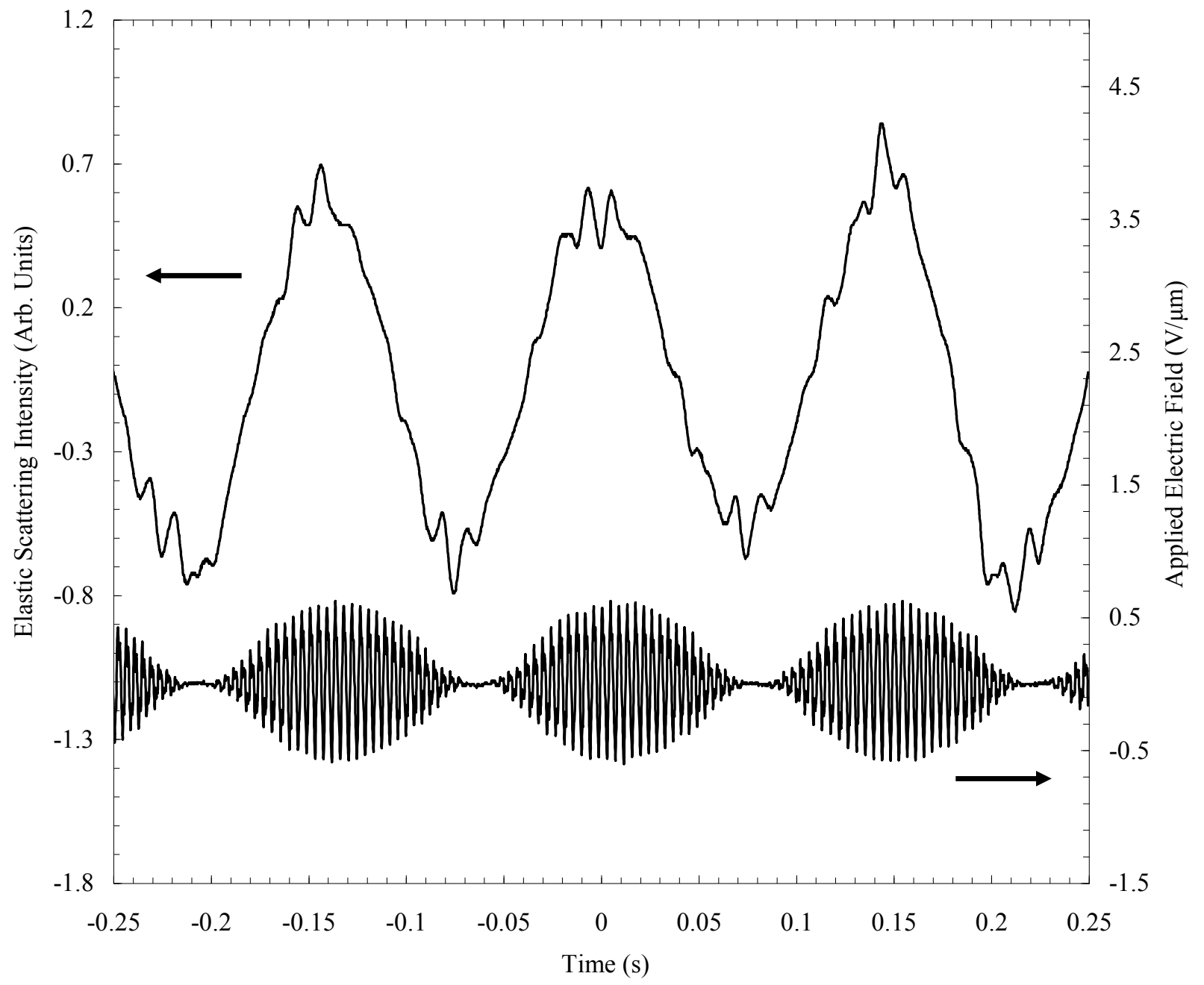

Figure 4. (upper curve) the modulated $90^{\circ}$ elastic scattering intensity, (lower curve) the modulating applied AC electrical field.

\section{CONCLUSIONS}

The MDR's of a silicon microsphere placed in a NLC and on an OFHC are excited with a tunable DFB semiconductor diode laser operating at $1.3 \mu \mathrm{m}$. By controlling the relative refractive index of the silicon microsphere, its MDR's are tuned and modulated in the transmission and $90^{\circ}$ elastic scattering signals. The control of the microsphere MDR's in this liquid crystal on silicon (LCOS) geometry has potential applications in photonics areas such as liquid crystal displays (LCD's).

\section{ACKNOWLEDGEMENTS}

We would like to acknowledge the partial support of this research by Türkiye Bilimsel ve Teknolojik Araştırma Kurumu (TÜBİTAK) grant No. EEEAG-106E215. 


\section{REFERENCES}

[1] K. J. Vahala, "Optical Microcavities,” Nature 424, 839 (2003).

[2] G. Griffel, S. Arnold, D. Taşkent, A. Serpengüzel, J. Connolly and N. Morris, "Morphology-dependant resonances of microsphere-optical fiber system,” Opt. Lett. 21, 695 (1996).

[3] F. Vollmer, D. Braun, A. Libchaber, M. Khoshsima, I. Teraoka and S. Arnold, "Protein detection by optical shift of resonant microcavity,” Appl. Phys. Lett. 80, 4057 (2002).

[4] A. Serpengüzel, A. Kurt and U. K. Ayaz, "Silicon microspheres for electronic and photonic integration," Photon. Nanostructur. Fundam. Appl. 6, 179 (2008)

[5] A. Serpengüzel, S. Arnold, and G. Griffel, "Excitation of Resonances of Microspheres on an Optical Fiber," Opt. Lett. 20, 654 (1995).

[6] A. Serpengüzel, S. Arnold, G. Griffel and J. A. Lock, "Enhanced coupling to microsphere resonances with optical fibers," J. Opt. Soc. Am. B 14, 790 (1997)

[7] Lord Rayleigh, "The problem of whispering gallery," Scientific Papers by Cambridge University 5, 617 (1912)

[8] C. F. Bohren and D. R. Huffman, [Adsorption and Scattering of Light by Small Particles], Wiley Science Paperback Series, New York, 100 (1983).

[9] T. Ioppolo, M. Kozhevnikov, V. Stepaniuk, M. V. Ötügen, and V. Sheverev, "Micro-Optical Force Sensor Concept Based on Whispering Gallery Mode Resonators," Appl. Opt. 47, 3009 (2008).

[10] C.-H. Dong, L. He, Y.-F. Xiao, V. R. Gaddam, S. K. Özdemir, Z.-F. Han, G.-C. Guo, and L. Yang, "Fabrication of High-Q Polydimethylsiloxane Optical Microspheres for Thermal Sensing,” Appl. Phys. Lett. 94, 231119 (2009).

[11]E. Yüce, O. Gürlü, and A. Serpengüzel, "Optical Modulation with Silicon Microspheres," IEEE Photon. Technol. Lett. 21, 1481 (2009).

[12] S. Arnold, M. Khoshsima, I. Teraoka, S. Holler, and F. Vollmer, "Shift of Whispering Gallery Modes in Microspheres by Protein Adsorption,” Opt. Lett. 28, 272 (2003).

[13] P. G. de Gennes and J. Prost, [The Physics of Liquid Crystals], Clarendon Press, Oxford, (1993). 